\title{
Response of nucleons to external probes in hedgehog models: I. Electromagnetic polarizabilities
}

\author{
Wojciech Broniowskit and Thomas D. Cohen \\ Department of Physics and Astronomy, University of Maryland \\ College Park, Maryland 20742-4111
}

\begin{abstract}
Electromagnetic polarizabilities of the nucleon are analyzed in a hedgehog model with quark and meson degrees of freedom. Semiclassical methods are used (linear response theory, quantization via cranking). It is found that in hedgehog models (Skyrmion, chiral quark models, Nambu-Jona-Lasinio model), the average electric polarizability of the nucleon, $\alpha_{N}$, is of the order $N_{c}$, and the splitting of the neutron and proton electric(proper) polarizabilities, $\delta \alpha=\alpha_{n}-\alpha_{p}$, is of the order $1 / N_{c}$. We present a general argument why one expects $\delta \alpha>0$ in models with a pionic cloud. Our model prediction for the sign and magnitude of $\delta \alpha$ is in agreement with recent measurements. The obtained value for $\alpha_{N}$, however, is roughly a factor of three too large. This is because of two problems with our particular model: a too strong pion tail, and the degeneracy of $N$ and $\Delta$ states in the large- $N_{c}$ limit. This degeneracy also results in a very strong $N_{c^{-}}$dependence of the paramagnetic part of the magnetic polarizability, $\beta$, which is of the order $N_{c}^{3}$. We compare the large- $N_{c}$ results to the one-loop chiral perturbation theory predictions, and show the importance of $\Delta$-effects in pionic loops. We also investigate the role of non-minimal substitution terms in the effective lagrangian on the polarizabilities of the nucleon.
\end{abstract}

PACS numbers: 12.38.Lg, 12.40.Aa, 14.20.Dh, 14.60.Fz

Typeset Using REVTEX 


\section{INTRODUCTION}

Polarizabilities are important fundamental properties of particles - they determine dynamical response of a bound system to external perturbations, and provide valuable insight into internal strong-interaction structure. Recent measurements of electromagnetic polarizabilities of the proton [1,2] and neutron [3] renewed theoretical interest in these quantities [4]. Many calculations of the electric, $\alpha$, and magnetic, $\beta$, polarizabilities of the nucleon can be found in the literature in models ranging from various quark models and bags [5 9] cloudy bags [10] to Skyrmions [11-13]. A quenched lattice calculation for $\alpha$ has also been done [14]. Extensive reviews of the subject are available [15.16].

In this paper we analyze the nucleon polarizabilities in hedgehog models, using the framework of the linear response theory. Our analysis differs significantly from previous works by allowing the soliton to deform ( dispersive effects). Our semiclassical methods are described in detail in the following paper [17], referred to as (II). The reader who is not familiar with the basics of hedgehog models or semiclassical methods used in their treatment, is urged to read (II) before this paper.

In recent years numerous hedgehog models, such as the Skyrme model [18 21], chiral quark models [22 27], hybrid bag models [28], chiral models with confinement [26, 29 31], or the Nambu-Jona-Lasinio model [32] in the solitonic treatment [33] 39], were quite successful in describing the phenomenology of nucleon structure. The basis for these hedgehogs models is the large- $N_{c}$ limit 40,41] of QCD. Thus, $1 / N_{c}$ should consistently be treated as an expansion parameter in this approach. Previous analyses of polarizabilities in hedgehog models [11 13 have not emphasized the need for consistent $N_{c}$-counting. In particular, a frozen approximation in the treatment of the response to electromagnetic perturbations was made, and the effects of distortions of the soliton, which occur at a relevant $N_{c}$-level, were not included. In this paper we develop consistent $N_{c}$-counting rules for $\alpha$ and $\beta$, and evaluate these observables in a specific model. We point out serious difficulties arising in the case of the magnetic polarizabilities. Special attention is paid to the issue of neutron-proton splitting of the polarizabilities, which has already been reported elsewhere [42].

Throughout this paper we use the so-called proper (or static) polarizabilities, $\alpha$ and $\beta$, defined via the energy shift of an object in constant electric, $E$, or magnetic, $H$, fields [43]:

$$
\delta \mathcal{E}=-\frac{1}{2} \alpha E^{2}-\frac{1}{2} \beta H^{2} .
$$

These are directly related (see Sec. [IIF) to the coefficients $\bar{\alpha}$ and $\bar{\beta}$ encountered in the Compton amplitude 44, 47, 15, 16,12

$$
\bar{\alpha}=\alpha+\Delta \alpha, \Delta \alpha=\frac{Q^{2}\left\langle r^{2}\right\rangle_{E}}{3 M}, \bar{\beta}=\beta,
$$

where the recoil term $\Delta \alpha$ involves the charge, $Q$, mass $M$, and electric mean square radius of the particle. Although the experimental errors are still substantial [48], the new measurements indicate that the proper electric polarizability is larger for the neutron than for the proton,

$$
\begin{aligned}
& \alpha_{n}=(12.0 \pm 1.5 \pm 2.0) \times 10^{-4} \mathrm{fm}^{3} \\
& \alpha_{p}=(7.2 \pm 1.0 \pm 1.0) \times 10^{-4} \mathrm{fm}^{3}
\end{aligned}
$$


and that the magnetic polarizabilities are positive, and equal within experimental errors:

$$
\begin{aligned}
& \beta_{n}=(3.1 \mp 1.5 \mp 2.0) \times 10^{-4} \mathrm{fm}^{3}, \\
& \beta_{p}=(3.4 \mp 1.0 \mp 1.0) \times 10^{-4} \mathrm{fm}^{3} .
\end{aligned}
$$

A number of results presented in this paper are generic to any hedgehog model, e.g. the $N_{c}$-counting rules and the role of the intermediate $\pi-\Delta$ states. Our numerical results are obtained using the simple model of Ref. [22] (Sec. II of (II)). Since this model possesses both quark and meson degrees of freedom, it is in this respect representative of a general class of hedgehog models. Our calculations can be repeated in any hedgehog model with minor modifications.

The organization of this article is as follows: In Sec. III we write down the gauged $\sigma$ model lagrangian, introduce collective coordinates in the usual way (Sec. III F of (II)), and classify various electromagnetic perturbations according to the hedgehog symmetries. We discuss the sea-gull and dispersive contributions to the Compton amplitude (Sec. IIB), and present the $N_{c}$-counting rules (Sec. IID). For the electric polarizabilities we find that the neutron-proton splitting effect is two powers of $N_{c}$ suppressed compared to the average nucleon value:

$$
\begin{gathered}
\alpha_{N}=\left(\alpha_{n}+\alpha_{p}\right) / 2 \sim N_{c}, \\
\delta \alpha=\alpha_{n}-\alpha_{p} \sim 1 / N_{c} .
\end{gathered}
$$

For the magnetic case the $N_{c}$-counting is more complicated (Sec. [V]).

In Sec. III we obtain numerical results for the electric polarizabilities. For $\alpha_{N}$, the seagull contribution (Sec. [IIA) is dominant, and the valence quark effects enter at the level of $10 \%$. This is due to the long-range nature of the pion. Our model prediction is about a factor of three too large than the experimental number. We discuss this discrepancy, which is due to two resons. Firstly, our specific model has a pionic tail which is too strong (i.e. the value of $g_{\pi N N}$ is too large compared to nature). This enhances the model prediction by about a factor of 2. A more fundamental reason is discussed in Sec. $\mathrm{V}$, where we point out that hedgehog predictions for some observables largely overestimate results due to the implicit treatment of the $\Delta$ resonanse as degenerate with the nucleon. Our prediction for $\delta \alpha=\alpha_{n}-\alpha_{p}$ are less sensitive on the strength of the pionic tail, and they are not affected by problems discussed in Sec. $\nabla$. Numerically, we obtain $\delta \alpha=5.4 \times 10^{-4} \mathrm{fm}^{3}$ [42, in agreement with the sign and magnitude given by the experiment. This splitting arises naturally in hedgehog models when dispersive effects are taken into account. The effect is dominated by the distortion of the pionic cloud. We also present a classical argument why the sign of $\delta \alpha$ is expected to be positive in models with pionic clouds (Sec. IIIE).

In Sec [IIF, we show that the standard retardation correction (Eq. 1.2) strictly holds in our linear response method. In Sec. IV we discuss the issue of magnetic polarizability in hedgehog models. We show that the degeneracy of the $\Delta$ and nucleon masses in the large- $N_{c}$ limit precludes the use of linear response theory to determine $\beta$. The $N-\Delta$ paramagnetic contribution, $\beta_{N \Delta}$ (given by the Born term with the intermediate $\Delta$ state) dominates the $N_{c}$ behavior of $\beta$, and leads to

$$
\beta_{N}=\left(\beta_{n}+\beta_{p}\right) / 2 \sim N_{c}^{3},
$$


In our view, this invalidates the claims of Refs. [11 13] that the diamagnetic sea-gull interaction cancels the paramagnetic $\Delta$ term, since these terms occur at different $N_{c}$-levels In Sec. $\mathbb{\nabla V}$ we also show the relevance of dispersive contributions in the calculation of the magnetic polarizability.

In Sec. $\nabla$ we compare our results to predictions of the chiral perturbation theory $(\chi P T)$. We find, that in the chiral limit the hedgehog predictions and the $\chi P T$ predictions for $\alpha$ and $\beta$ agree up to a factor which can be attributed to the role of the $\Delta$ in pionic loops. We discuss interesting physical implications of this issue, and point out that while the naive hedgehog predictions overestimate the role of the $\Delta$ resonance in hadronic loops, the naive approach to $\chi P T$ drops these important contributions altogether. We discuss the effects of finite $N-\Delta$ mass splitting in these approaches, and propose how the hedgehog and $\chi P T$ results should be corrected.

In Sec. VI we study the effects of the pionic substructure on nucleon polarizabilities.

These effects are introduced via non-minimal substitution terms $\mathcal{L}_{9}$ and $\mathcal{L}_{10}$ [49,50] in the effective lagrangian. At the mean-field level, we obtain simple expressions involving pion polarizability and pion mean squared radius. Numerically, these pion structure contributions enter at the level of $\sim 1-2 \times 10^{-4} \mathrm{fm}^{3}$, which is a small but non-negligible effect.

In Sec. VII we make a few comments relevant to calculations in other hedgehog models. Throughout the paper we use the units $\hbar=1, c=1, e^{2} /(\hbar c)=1 / 137$.

\section{ELECTROMAGNETIC PERTURBATIONS}

Our basic methods of treatment of external perturbations in hedgehog models are described in (II). The approach developed in (II) is a rather straightforward adaptation of the RPA method of traditional many-body physics. The diference with the traditional nuclear physics case is the presence of mesonic degrees of freedom, relativistic dynamics, and special symmetries (Sec. II B in (II)).

Polarizabilities are defined as second-order energy shifts due to (static) external electromagnetic fields, Eq. (1.1), or, equivalently, via Compton amplitude (Sec. IIB). They arise from two types of terms: sea-gulls, which are due to quadratic terms in $E$ or $B$ in the lagrangian, and from dispersive terms. Sea-gull effects result in local expressions involving the pion mean field [11 13], while the dispersive terms are given by the usual second-order perturbation theory expressions, which result from linear perturbations in the lagrangian. These dispersive effects distort the soliton. They are particularly important in the neutronproton splitting effects, as well as in the magnetic response, and have not been considered in earlier works. More importantly, these effects enter at the same $N_{c}$-level as the sea-gull terms, and thus should be included in order to comply with the basic organizational principle of the hedgehog approach, namely, the $1 / N_{c}$-perturbation expansion. The dispersive terms in the Compton amplitude correspond, in our approach, to diagrams in which the propagator between the two photon insertions is the RPA (or linear response) propagator (Fig. 11. Relevant expressions are obtained using equations-of-motion method, which amounts to solving linear differential equations with potentials and driving terms determined by the solitonic profile. In addition, electromagnetic perturbations are carried in the presence of cranking, which ensures projection on states with good quantum numbers (Sec. II G in (II)). 


\section{A. Lagrangian in presence of electromagnetic interactions}

The first step is to identify perturbations resulting from coupling to electromagnetic interactions, which are introduced by gauging the $\sigma$-model lagrangian (II.2.1):

$$
\begin{aligned}
\mathcal{L} & =\bar{\psi}\left[i \not \partial+g\left(\sigma+i \gamma_{5} \boldsymbol{\tau} \cdot \boldsymbol{\pi}\right)\right] \psi \\
& +\frac{1}{2}\left(\partial^{\mu} \sigma\right)^{2}+\frac{1}{2}\left(\partial^{\mu} \boldsymbol{\pi}\right)^{2}-U(\sigma, \boldsymbol{\pi}) .
\end{aligned}
$$

Through this procedure one generates a dispersive interaction, as well as a covariantizing sea-gull term:

$$
\begin{aligned}
& \mathcal{L}_{\text {disp. }}=-e A^{\mu}\left(\bar{\psi}\left(\frac{1}{2 N_{c}}+\frac{1}{2} \tau_{3}\right) \gamma_{\mu} \psi+\left(\boldsymbol{\pi} \times \partial_{\mu} \boldsymbol{\pi}\right)_{3}\right), \\
& \mathcal{L}_{\text {sea-gull }}=\frac{1}{2} e^{2} A^{\mu} A_{\mu}\left(\boldsymbol{\pi}_{1}^{2}+\boldsymbol{\pi}_{2}^{2}\right),
\end{aligned}
$$

where $\psi$ is the quark field, $\boldsymbol{\pi}$ is the pion field, and $A^{\mu}$ is the photon field. It is hoped that this minimal substitution procedure gives the bulk of electromagnetic interactions in our effective hadronic lagrangian, however, non-minimal substitution terms may a priori play an important role. In Section $\nabla \mathbb{1}$ we discuss the role of simple non-minimal substitution terms in effective lagrangians, and find that their effects enter (in the electric polarizability) at the level of $10 \%$, compared to the terms resulting from (2.2).

To identify the appropriate perturbations, we make a transformation of the full lagrangian (II.2.1, 2.2) to the isorotating frame, according to Eqs. (II.3.22). This is done because we are interested in the linear response of the nucleon, which is "projected out" of the hedgehog (see Sec. III in (II) for details of projection via cranking, and linear response in presence of cranking). For a more compact notation, we also replace the quark field bilinears by $N_{c}$ times the bilinears of the valence quark, $q$. The Lorentz gauge is used, and $A^{0}=-\boldsymbol{r} \cdot \boldsymbol{E}, \boldsymbol{A}=-\frac{1}{2} \boldsymbol{r} \times \boldsymbol{B}$. We obtain

$$
\begin{aligned}
\mathcal{L} & \rightarrow \mathcal{L}^{0}+\mathcal{L}_{\lambda}^{1}+\mathcal{L}_{\lambda}^{2}+\mathcal{L}_{E^{0}}^{1}+\mathcal{L}_{E^{1}}^{1}+\mathcal{L}_{E^{1}}^{2}+\mathcal{L}_{\lambda E^{1}}^{2} \\
& +\mathcal{L}_{B^{0}}^{1}+\mathcal{L}_{B^{1}}^{1}+\mathcal{L}_{B^{1}}^{2} \\
\mathcal{L}^{0} & =N_{c} \bar{q}\left(i \not \partial+g\left(\sigma+i \gamma_{5} \boldsymbol{\tau} \cdot \boldsymbol{\pi}\right)\right) q \\
& +\frac{1}{2}\left(\partial^{\mu} \sigma\right)^{2}+\frac{1}{2}\left(\partial^{\mu} \boldsymbol{\pi}\right)^{2}-U(\sigma, \boldsymbol{\pi}), \\
\mathcal{L}_{\lambda}^{1} & =-N_{c} q^{\dagger}\left(\frac{1}{2} \boldsymbol{\lambda} \cdot \boldsymbol{\tau}\right) q-\boldsymbol{\lambda} \cdot(\boldsymbol{\pi} \times \dot{\boldsymbol{\pi}}) \\
\mathcal{L}_{\lambda}^{2} & =\frac{1}{2}(\boldsymbol{\lambda} \times \boldsymbol{\pi})^{2} \\
\mathcal{L}_{E^{0}}^{1} & =e \boldsymbol{r} \cdot \boldsymbol{E} \frac{1}{2} q^{\dagger} q \\
\mathcal{L}_{E^{1}}^{1} & =e \boldsymbol{r} \cdot \boldsymbol{E}\left(N_{c} q^{\dagger} \frac{1}{2} \boldsymbol{c} \cdot \boldsymbol{\tau} q+\boldsymbol{c} \cdot(\boldsymbol{\pi} \times \dot{\boldsymbol{\pi}})\right) \\
\mathcal{L}_{E^{1}}^{2} & =\frac{1}{2} e^{2}(\boldsymbol{r} \cdot \boldsymbol{E})^{2}(\boldsymbol{c} \times \boldsymbol{\pi})^{2} \\
\mathcal{L}_{\lambda E^{1}}^{2} & =-e \boldsymbol{r} \cdot \boldsymbol{E}(\boldsymbol{c} \times \boldsymbol{\pi}) \cdot(\boldsymbol{\lambda} \times \boldsymbol{\pi}),
\end{aligned}
$$




$$
\begin{aligned}
\mathcal{L}_{B^{0}}^{1} & =-\frac{1}{4} e(\boldsymbol{r} \times \boldsymbol{B})^{i} q^{\dagger} \alpha_{i} q, \\
\mathcal{L}_{B^{1}}^{1} & =-\frac{1}{2} e(\boldsymbol{r} \times \boldsymbol{B})^{i}\left(N_{c} q^{\dagger} \frac{1}{2} \boldsymbol{c} \cdot \boldsymbol{\tau} \alpha_{i} q\right. \\
& \left.-\boldsymbol{c} \cdot\left(\boldsymbol{\pi} \times \nabla_{i} \boldsymbol{\pi}\right)\right) \\
\mathcal{L}_{B^{1}}^{2} & =-\frac{1}{8} e^{2}(\boldsymbol{r} \times \boldsymbol{B})^{2}(\boldsymbol{c} \times \boldsymbol{\pi})^{2},
\end{aligned}
$$

where $\boldsymbol{\lambda}$ is the cranking velocity, and the collective vector $\boldsymbol{c}=\frac{1}{2} \operatorname{Tr}\left[\tau_{3} B \boldsymbol{\tau} B^{\dagger}\right]$ is defined in App. B in (II). In the above equations, subscript $\lambda$ refers to term arising upon cranking, $E^{0}$ and $E^{1}$ denote isoscalar and isovector electric perturbations, and $B^{0}$ and $B^{1}$ denote isoscalar and isovector magnetic perturbations. Indices $i$ in Eqs. 2.11 - 2.12 are spatial Cartesian indices. Superscript ${ }^{1}$ denotes dispersive terms, which lead to linear shifts in fields according to perturbation theory. Superscript ${ }^{2}$ denotes terms which are quadratic in perturbations. They include a term quadratic in $\lambda$ (Eq. 2.6), sea-gull terms (Eqs. 2.9,2.13), and a mixed term (Eq. 2.10), which will play an essential role in the splitting of the neutron and proton polarizabilities. The classification of various dispersive terms, as well as the explicit forms of the corresponding sources arising in linear response equations, are given in Table $\mathbb{1}$. The grand spin (sum of spin and isospin) is denoted by $K$, parity is denoted by $\mathcal{P}$, and the grand-reversal symmetry by $\mathcal{R}$ (see Sec. II B in (II) for details).

\section{B. Compton amplitude and polarizabilities}

The Compton amplitude corresponding to lagrangian (2.3) reflects the presence of both dispersive and sea-gull terms. It can be written as 12

$\tilde{M}_{\mu \nu}(p, q)=M_{\mu \nu}(p, q)+S_{\mu \nu}(q)$,

$M_{\mu \nu}(p, q)=$

$i \int d^{4} x e^{i q \cdot x}\left\langle N\left(p^{\prime}\right)\left|T\left(J_{\mu}^{e . m \cdot}(x) J_{\nu}^{e . m \cdot}(0)\right)\right| N(p)\right\rangle$,

where $M_{\mu \nu}$ is the dispersive $T$-product part, and $S_{\mu \nu}$ is the sea-gull part. Correspondingly, the polarizabilities have dispersive as well as sea-gull parts, and can be written as 12

$$
\begin{aligned}
\alpha & =\alpha_{\text {disp. }}+\alpha_{\text {sea-gull }}, \quad \beta=\beta_{\text {disp. }}+\beta_{\text {sea-gull }}, \\
\alpha_{\text {disp. }} & =2 \sum_{b^{\prime}} \frac{\left|\left\langle N\left|\int d^{3} x \widehat{\boldsymbol{E}} \cdot \boldsymbol{r} J_{0}^{e . m .}(\boldsymbol{r})\right| b^{\prime}\right\rangle\right|^{2}}{E_{b^{\prime}}-E_{N}}, \\
\alpha_{\text {sea-gull }} & =\left\langle N\left|\int d^{3} x(\widehat{\boldsymbol{E}} \cdot \boldsymbol{r})^{2} S_{00}\right| N\right\rangle, \\
\beta_{\text {disp. }} & =2 \sum_{b^{\prime}} \frac{\left|\left\langle N\left|\int d^{3} x \frac{1}{2} \widehat{\boldsymbol{B}} \cdot\left(\boldsymbol{r} \times \boldsymbol{J}^{\text {e.m. }}(\boldsymbol{r})\right)\right| b^{\prime}\right\rangle\right|^{2}}{E_{b^{\prime}}-E_{N}}, \\
\beta_{\text {sea-gull }} & =\frac{1}{4}\left\langle N\left|\int d^{3} x \epsilon_{m n i} \widehat{B}^{m} r^{n} \epsilon_{k l j} \widehat{B}^{k} r^{l} S_{i j}\right| N\right\rangle .
\end{aligned}
$$

Hats denote unit vectors in the direction of $E$ or $B$ fields, and $\left|b^{\prime}\right\rangle$ is an intermediate state with energy $E_{b^{\prime}}$. 
The sea-gull parts of expressions (2.15) can be readily identified from Eq. 2.9,2.13). One gets

$$
\begin{aligned}
& S_{00}=e^{2}(\boldsymbol{c} \times \boldsymbol{\pi})^{2}, \\
& S_{i j}=-e^{2} \delta_{i j}(\boldsymbol{c} \times \boldsymbol{\pi})^{2} .
\end{aligned}
$$

Evaluating these in the collective nucleon state one obtains (App. B in (II), [12,13])

$$
\alpha_{\text {sea-gull }}=-2 \beta_{\text {sea-gull }}=\frac{8 \pi e^{2}}{9} \int d r r^{4} \pi_{h}^{2}
$$

where $\pi_{h}$ is the hedgehog pion field profile. In Eq. (2.17) we recognize the hedgehog model relation between the seagull contributions to the electric and magnetic polarizabilities 12, 13. The radial integral in Eq. (2.17) is quadratic in the pion field. In the Skyrme model additional terms arise due to higher-order terms in the lagrangian [11 [13].

\section{Linear response}

In our treatment of dispersive contributions, the intermediate states $\left|b^{\prime}\right\rangle$ in Eq. (2.15) correspond to RPA excited states, and the energy denominators involve energies of these excitations (Fig. 1). These contributions can be written in the general form given in Eqs. (II.3.27). The electric and magnetic dispersive polarizabilities result from either isoscalar dipole or isovector dipole transitions, and will be labeled by $E^{0}$ and $E^{1}$, or $B^{0}$ and $B^{1}$, respectively. These dispersive contributions, as well as the sea-gull contributions (2.17) contribute equal amounts to the neutron and the proton. This is because the resulting collective operators in Eqs. (II.3.27) are isoscalar (bilinear in the collective vector $\boldsymbol{c}$ ), and matrix elements are equal for the proton and the neutron. The neutron-proton splitting effects arise from the mechanism described in Sec. III G in (II). This can be briefly described in the following way: We perform electromagnetic perturbations not on hedgehogs, but on nucleons. These are obtained from hedgehogs via projection (cranking), which in turn may also be viewed as linear response. It is sufficient to work to linear order in the cranking velocity, $\boldsymbol{\lambda}$, and we can write down symbolically

$$
|N\rangle=\left(1+\mathcal{G} \mathcal{V}_{c r}\right)|H\rangle
$$

where $\mathcal{G}$ is the RPA propagator, $\mathcal{V}_{c r}$ is the cranking interaction, and $|H\rangle$ is the hedgehog state. Using these "cranked" states in our perturbation theory leads to expressions of the form of Eqs. (II.3.29), with one cranking, and two electromagnetic interactions. As discussed in Sec. III G in (II), the $K^{\mathcal{P}}$ numbers of interactions in Eqs. (II.3.29) have to be additive to $K^{\mathcal{P}}=0^{+}$, since the hedgehog has $K^{\mathcal{P}}=0^{+}$. From Table $\mathbb{\|}$ we can see that we can compose cranking, one isoscalar electric (magnetic), and one isovector electric (magnetic) interaction to $K^{\mathcal{P}}=0^{+}$. Since $\mathcal{V}_{c r}$ carries a collective operator $\lambda$, the appropriate collective operator is isovector, and neutron-proton splitting of polarizabilities is generated.

From a slightly different but equivalent point of view, we may understand the isospin effect in electric polarizabilities by inspecting the term (2.10). It is linear in cranking, and linear in isovector electric $\left(E^{1}\right)$ perturbation. It leads to the following contribution to $\alpha$ : 
$\alpha_{\lambda}^{m e s .}=-e\left\langle N\left|\int d^{3} r(\widehat{\boldsymbol{E}} \cdot \boldsymbol{r})[(\boldsymbol{c} \times \boldsymbol{\pi}) \cdot(\boldsymbol{\lambda} \times \boldsymbol{\pi})]\right| N\right\rangle$.

If the pion field in the above equation were taken to be just the hedgehog profile, then $\alpha_{\lambda}^{\text {mes. }}$ would vanish by parity. However, the isoscalar electric perturbation (Section [II ) distorts the meson fields, and $\boldsymbol{\pi}=\boldsymbol{\pi}_{h}+\delta \boldsymbol{\pi}_{E^{0}}$, where $\delta \boldsymbol{\pi}_{E^{0}}$ has $S$ - and $D$-wave components. As a result, the integral is non-zero. The corresponding collective operator is $\boldsymbol{c} \cdot \boldsymbol{\lambda}=I_{3} / \Theta$, where $\Theta$ is the moment of inertia (see App. B in (II)), and we obtain

$\alpha_{\lambda}^{m e s .}=-e \frac{I_{3}}{\Theta} 8 / 3 \int d r(\widehat{\boldsymbol{E}} \cdot \boldsymbol{r})\left(\boldsymbol{\pi}_{h} \cdot \delta \boldsymbol{\pi}_{E^{0}}\right),$.

From the quark parts of Eqs. (2.3) we get

$$
\begin{aligned}
\alpha_{\lambda}^{q u a r k} & =2\langle N| \int d^{3} r\left[-\delta q_{E^{0}}^{\dagger} \frac{1}{2} \boldsymbol{\lambda} \cdot \boldsymbol{\tau} \delta q_{E^{1}}+\delta q_{E^{1}}^{\dagger} \frac{e \widehat{\boldsymbol{E}} \cdot \boldsymbol{r}}{2 N_{c}} \delta q_{\lambda}\right. \\
& \left.+\delta q_{E^{0}}^{\dagger} \frac{1}{2} e \widehat{\boldsymbol{E}} \cdot \boldsymbol{r c} \cdot \boldsymbol{\tau} \delta q_{\lambda}\right]|N\rangle+h . c .
\end{aligned}
$$

where various terms in the interaction are sandwiched with shifts corresponding to other interactions. Eqs. (2.20,2.21) can be straightforwardly obtained from Eq. (II.3.29). Terms $(2.20,2.21)$ are proportional to the nucleon isospin, $I_{3}$, thus are responsible for splitting of the neutron and proton electric polarizabilities, $\delta \alpha$.

For the splitting of magnetic polarizabilities, mesons do not contribute at the linearresponse level, since there is no mesonic analog of the term (2.10) in lagrangian (2.3). Only the magnetic analog of the quark part (Eq. 2.21) is present. However, the linear response calculation of the magnetic polarizability encounters fundamental problems, which will be discussed in Sec. [V.

\section{D. $N_{c}$ - counting}

In Eqs. 2.3 we list explicitly occurrences of $N_{c}$. We also recall [41] that in the large- $N_{c}$ limit the meson fields scale as $\sqrt{N_{c}}$, the quark fields scale as 1 , and the moment of inertia scales as $N_{c}$. Note that the sources for isoscalar electromagnetic perturbations are one power down compared to the corresponding sources for the isovector interactions. We easily arrive at the following $N_{c}$-rules for electric polarizabilities:

$\alpha_{\text {sea-gull }} \sim N_{c}, \quad \alpha_{E^{0}} \sim 1 / N_{c}, \quad \alpha_{E^{1}} \sim N_{c}, \quad \alpha_{\lambda} \sim 1 / N_{c}$.

This leads directly to the following rules for the nucleon polarizabilities:

$$
\begin{aligned}
\alpha_{N} & =\frac{\alpha_{n}+\alpha_{p}}{2}=\alpha_{\text {sea-gull }}+\alpha_{E^{1}} \sim N_{c}, \\
\delta \alpha & =\alpha_{n}-\alpha_{p}=\alpha_{\lambda} \sim 1 / N_{c} .
\end{aligned}
$$

Note, that since the $\alpha_{E^{0}}$ part of the dispersive term contributes to $\alpha_{N}$ at a subleading level, it should be dropped according to $N_{c}$-rules. Other physical effects affect our results at this level, e.g. centrifugal stretching, center-of-mass corrections to the soliton mass, $N_{c}$-suppressed terms in the effective lagrangian, etc. Numerically, the $\alpha_{E^{0}}$ contribution is negligible, confiring the validity of $N_{c}$-counting for polarizabilities.

Naively, one would write down expressions analogous to Eqs. (2.23) for the magnetic polarizability, $\beta$. We will show in Sec. IV that this is not correct. 


\section{ELECTRIC POLARIZABILITY}

In this section we present our numerical results for $\alpha$. Numerical methods, explicit forms of the differential equations, and other details are given in (II). The model parameters for our numerical calculations are taken from Refs. 22, 51]: $F_{\pi}=93 \mathrm{MeV}, g F_{\pi}=500 \mathrm{MeV}$, $m_{\pi}=139.6 \mathrm{MeV}$, and $m_{\sigma}=1200 \mathrm{MeV}$.

\section{A. Sea-gull contribution}

Because the pion field is long ranged, the nucleon electric polarizability, $\alpha_{N}$, is dominated by the pionic sea-gull contribution (2.17). Numerically, in our model we get

$$
\alpha_{\text {sea-gull }}=28 \times 10^{-4} \mathrm{fm}^{3},
$$

which is roughly three times larger than the experimental value in (1.3). There are two basic reasons for this discrepancy. The first one is discussed below, and the other in Sec. D. Figure (2) shows the radial density of the integrand of (2.17) (solid line). It is clear that the sea-gull term acquires most of its value from the asymptotic region, $r>1 \mathrm{fm}$. Recall, that the asymptotic behavior of the pion tail in hedgehog models is determined by the pion-nucleon coupling constant, $g_{\pi N N}$ [20,51]. Using the Goldberger-Treiman relation, we can write down

$$
\boldsymbol{\pi}_{h}^{\text {asympt. }}=\left(3 g_{A}\right) /\left(8 \pi F_{\pi}\right) \hat{\boldsymbol{x}}\left(m_{\pi}+1 / r\right) \exp \left(-m_{\pi} r\right) / r .
$$

Our model predicts $g_{A}=1.86$ [51], hence the tail contribution is overestimated by a factor of $\left(g_{A}^{\text {model }} / g_{A}^{\text {exp. }}\right)^{2} \sim 2$. The dotted line in Fig. (2) shows the integrand of Eq. (2.17) with the pion field having the form (3.2) in the whole region of $r$. This corresponds to the chiral limit case, and will be discussed in detail in Sec. D. We can see from Fig. (2) that the size of $\alpha_{\text {sea-gull }}$ is controlled by how fast the solid line departs from the dashed line at lower values of $r$. In other words, the results are sensitive to the profile of the pion field in the intermediate region of about $1 \mathrm{fm}$. Note, that $\pi_{h}$ enters the expression (2.17) quadratically. Therefore, we expect substantial model sensitivity in hedgehog predictions of $\alpha_{\text {sea-gull }}$, e.g. models which have a suppressed pion field, such as hedgehog models with confinement [26, 29] 31], or models with vector mesons, e.g. [52,53], are expected to predict lower values for $\alpha_{\text {sea-gull }}$. We stress that to compare fairly the model predictions with experiment, the model should predict correctly the quantity $g_{A} / F_{\pi}$, which enters the asymptotic form (3.2).

In Sec. $\square$ we show how the sea-gull contribution is additionally suppressed when $N-\Delta$ mass splitting effect is taken into account. This results in another factor of $\sim 2$ reduction. With these corrections we note that it is possible to put the sea-gull contribution in the right experimental range. The model uncertainty, however, is big.

\section{B. Isovector electric perturbation}

In Sec. IID we have shown that the dispersive electric isovector $\left(E^{1}\right)$ contribution to polarizability, $\alpha_{E^{1}}$, is of the order $N_{c}$, the same order as for $\alpha_{\text {sea-gull}}$. The $E^{1}$ perturbation 
in lagrangian (2.8) has $K^{\mathcal{P R}}=0^{--}, 1^{--}$and $2^{--}$(Table 四). Since it is odd under grandreversal $\mathcal{R}$, only the valence quark fields acquire shifts (Sec. III A in (II)), and we solve equations of the form of Eqs. (II.3.7):

$$
(h-\varepsilon) q_{E^{1}}=\frac{1}{2} N_{c} e \boldsymbol{r} \cdot \widehat{\boldsymbol{E}} \boldsymbol{c} \cdot \boldsymbol{\tau} q_{h} .
$$

These equations are decomposed into $K=0,1$ and 2 components, as described in App. A in (II), and solved numerically. The corresponding polarizability is calculated according to the expression

$$
\alpha_{E^{1}}=-2 N_{c} e\left\langle N\left|\int d^{3} x q_{E_{1}}^{\dagger} \boldsymbol{r} \cdot \widehat{\boldsymbol{E}} \boldsymbol{c} \cdot \boldsymbol{\tau} q_{h}\right| N\right\rangle,
$$

where $|N\rangle$ is the nucleon collective state (App. B in (II)). Note that with our definition, the spinor $q_{E^{1}}$ carries the collective variable $\boldsymbol{c}$, such that Eq. (3.4) contains a matrix element of a collective operator quadratic in $\boldsymbol{c}$, similarly to the case of the sea-gull part in Sec. IIB.

Numerically, we get in our model

$$
\alpha_{E^{1}}=3.49 \times 10^{-4} \mathrm{fm}^{3},
$$

with $K=0,1$, and 2 components of $q_{E_{1}}$ contributing $-12 \%, 26 \%$, and $86 \%$, respectively. The dashed line in Fig. 2 depicts the radial density of $\alpha_{E^{1}}$. Because the quark mass in our model is $500 \mathrm{MeV}$, the purely quark $\alpha_{E^{1}}$ contribution is strongly suppressed compared to the pion sea-gull contribution. However, the value of $\alpha_{E^{1}}$ is non-negligible compared to experimental numbers (Table $\llbracket$ II), and quark effects are substantial.

In models with vector mesons [52,53, the $E^{1}$ perturbation involves shifts in the $\rho$ and $\omega$ mesons, and these effects must be included in calculations of the electric polarizability [54]. They enter at the leading- $N_{c}$ level, and should contribute comparably to the valence quark effects.

\section{Isoscalar electric perturbation}

Now we turn to a more difficult case of the isoscalar electric perturbation, $E^{0}$, which has $K^{\mathcal{P R}}=1^{-+}$. These are quantum numbers of the translational zero mode, and, as explained in Sec. III A in (II), we have to deal with full RPA equations of the form (II.3.3). The appearance of the zero mode is easy to understand. The hedgehog soliton possesses isoscalar electric charge, $Q^{I=0}=\frac{1}{2} e$. Thus, in a constant electric field, the soliton accelerates in the direction of $\boldsymbol{E}$ - the translational mode is excited. In addition to this zero-mode motion, the soliton is getting distorted (as viewed from the center of mass). Below we show that the excitation of the translational motion corresponds to the Thompson limit of the Compton scattering, and the deformation corresponds to the polarizability. In order to separate the zero mode from the physical modes, instead of a constant electric field we consider a slowly time-dependent field of the form

$$
E(t)=\frac{1}{2}\left(E_{0} e^{-i \omega t}+E_{0}^{*} e^{i \omega t}\right)
$$

The resulting sources in Eq. (II.3.3) are 


$$
\begin{aligned}
& j_{X}=j_{Y}=\frac{1}{2} e N_{c}^{-1}(\boldsymbol{r} \cdot \widehat{\boldsymbol{E}}) q_{h}, \\
& j_{Z}=j_{P}=0 .
\end{aligned}
$$

Using the technique described in Sec. III B in (II), we find that the total isoscalar electric polarizability consist of the translational zero-mode part, and a "physical" part:

$$
\alpha_{E^{0}}^{\text {tot. }}=\alpha_{E^{0}}^{z e r o}+\alpha_{E^{0}}^{\text {phys. }}
$$

where the zero-mode part diverges in the limit $\omega \rightarrow 0$,

$$
\alpha_{E^{0}}^{z e r o}=-\frac{\left(Q^{I=0}\right)^{2}}{M \omega^{2}}
$$

and the physical part, $\alpha_{E^{0}}^{\text {phys }}$, has a finite $\omega \rightarrow 0$ limit. The quantity $M$ in Eq. (3.9) is the soliton mass. In the expression for the forward Compton scattering amplitude, $\alpha$ is multiplied by $\omega^{2}$ 47,44,16]. We note immediately that $\omega^{2} \alpha_{E^{0}}^{z e r o}=-\frac{\left(Q^{I=0}\right)^{2}}{M}$ is just the Thompson term in scattering of a particle with charge $Q^{I=0}$ and mass $M$. Thus, the zeromode part is responsible for the Thompson limit. Since RPA leads to small fluctuation equations of motion, analogously to the case of classical physics [44, it is clear it leads to the correct Thompson limit.

In the case of the $E^{1}$ perturbation, the quantum numbers precluded excitation of a zeromode on top of the hedgehog solution. This is a manifestation of the fact that the hedgehog does not have any isovector electric charge, $Q^{I=1}=0$. This charge arises only upon cranking, but the organization of our perturbation theory is such, that we treat cranking, $E^{0}$ and $E^{1}$ perturbations separately (Sec. III G and V in (II)). If linear response were performed on a cranked soliton, then the full charge $Q=Q^{I=0}+Q^{I=1}$ would appear in Thompson scattering.

Earlier works [12,13 on electric polarizabilities in hedgehog models did not take into account the effects of zero modes. In fact, this is justified by the $1 / N_{c}$ expansion. The $E^{0}$ contribution is suppressed by two powers of $N_{c}$ compared to the $E^{1}$ contribution (Eq. (2.22). Therefore, if one is interested in the leading $N_{c}$ behavior of $\alpha_{N}$, it is sufficient to consider the isovector electric perturbation only, where the issue of the translational zero mode does not arise.

Since we are interested in the splitting of the neutron and proton polarizabilities, we have to calculate the quantities $\alpha_{\lambda}^{\text {mes. }}$ (Eq. (2.20)) and $\alpha_{\lambda}^{\text {quark }}$ (Eq. (2.21)). Thus, we have to find the shifts in the fields due to the isoscalar perturbation, $E^{0}$. We need to extract the "physical" parts of the solution. The numerical procedure has been described in Sec. III $\mathrm{C}$ in (II). Here we only remark, that very good numerical accuracy is necessary in order to separate the zero mode from the physical mode. This is because at small values of $\omega$ the solution can be written as

$$
\xi=\frac{i \dot{a t h} Q_{0}}{\mathcal{M} \omega^{2}} \xi_{0}-\frac{Q_{0}}{\mathcal{M} \omega} \xi_{1}+\xi_{\text {phys }}+\mathcal{O}(\omega),
$$

where $\xi_{0}$ is the translational mode, $\xi_{1}$ is the conjugated mode ("boost mode"), and $\xi_{\text {phys. }}$ is the physical mode which we want to extract (Sec. III B in (II)). Solutions of equations (II.3.3) in the limit $\omega \rightarrow 0$ give full $\xi$, from which we subtract the pieces divergent in $\omega$. This can easily be done, since we know the exact forms of $\xi_{0}$ and $\xi_{1}$ (App. C in (II)). The presence of the zero mode provides a useful algebraic check of equations from App. A in (II), since the divergent parts of Eq. (3.10) have known coefficients. 


\section{Neutron-proton splitting of electric polarizabilities}

Having solved the linear response equations for the $E^{0}$ perturbation, we use $q_{E^{0}}^{\text {phys. }}=$ $X^{\text {phys. }}+Y^{\text {phys }}$ and $\phi^{\text {phys. }}=2 Z^{\text {phys. }}($ App. A in (II)) in equations (2.20, 2.21). The dominant part to $\alpha_{\lambda}$ comes from the pionic contribution. The pion shift has $S$ - and $D$-wave components (Table 回), which we denote by $\pi_{S}$ and $\pi_{D}$, respectively. The explicit expression for the mesonic contribution of $\delta \alpha^{\text {mes }}$ has the form

$$
\delta \alpha^{m e s}=-\frac{16 e}{3 \Theta} \sqrt{\frac{4 \pi}{3}} \int d r r^{3} \pi_{h}\left(\sqrt{1 / 3} \pi_{S}-\sqrt{2 / 3} \pi_{D} .\right.
$$

The quark contribution is obtained analogously from Eq. (2.21). The numerical results give

$$
\delta \alpha=-5.6 \times 10^{-4} \mathrm{fm}^{3},
$$

with the quarks carrying $-5 \%$ of the total. Hence, as in the $E^{1}$ case, we observe the dominance of the pionic contribution. Figure 3 shows the radial density of expression (3.11). The long-range nature of the pion is evident. As discussed in Sec. IIIB, our predictions are enhanced due to the fact that $g_{A}$ has a too large value in our model. In the present case, however, we estimate this effect at the level of $g_{A}^{\text {model }} / g_{A}^{\text {exp. }} \sim 1.4$, rather than a factor of 2 in the pionic sea-gull term. This is because Eq. (3.11) is linear in $\pi_{h}$ (recall that asymptotically $\pi_{h}$ is proportional to $g_{A}$, Eq. (3.2)), and $\pi_{S}$ and $\pi_{D}$ are not dependent on the strength of the pionic tail in $\pi_{h}$. The reason is that the sources in the linear response equations come from the quarks, which are short-ranged. In the asymptotic region, where Eq. (3.11) gets most of its contribution, the shifts in the pion field, $\pi_{S}$ and $\pi_{D}$, depend on the total strength

of the source $\left(Q^{I=0}=\frac{1}{2}\right)$, and the pion mass, which enters the Green's function, but not on the strength of the tail of $\pi_{h}$. Consequently, our overestimate of $\delta \alpha$ should be at the level of $40 \%$ only.

Our results are summarized in Table $\mathbb{I}$. We note, that the sign of the neutron-proton splitting effect, and its magnitude, agree with the recent experimental numbers.

\section{E. Pionic cloud and sign of $\delta \alpha$}

In the previous section the sign of $\delta \alpha=\alpha_{n}-\alpha_{p}$ was found to be negative. The following plausibility argument can be given as to why we expect this behavior in models with pionic clouds. The hedgehog models imply that the nucleon consists of a quark core, carrying isoscalar charge, and a pion cloud, carrying isovector charge. In the proton, these charges have the same sign, and the electric field distorts both the core and the cloud, but it does not displace them relative to each other. In the case of the neutron, the cloud and the core, in addition to being deformed also get displaced, since their charges are opposite. This results in additional polarizability. Calculations based on other methods also give $\alpha_{n}>\alpha_{p}$ [55,56.

\section{F. Retardation term}

Our calculation was performed with a spatially constant electric field. Now consider plane-wave photons, as in Compton scattering, i.e. 


$$
\begin{aligned}
\boldsymbol{E}=\boldsymbol{E}_{0} \exp \left(i \boldsymbol{k}_{\perp} \cdot \boldsymbol{x}\right) & \simeq E_{0}\left(1+i \boldsymbol{k}_{\perp} \cdot \boldsymbol{x}-\frac{1}{2}\left(\boldsymbol{k}_{\perp} \cdot \boldsymbol{x}\right)^{2}\right), \\
k_{\perp}{ }^{2} & =\omega^{2}
\end{aligned}
$$

The linear-response source, $j$, is modified accordingly, and the charge $Q=\xi_{0}{ }^{\dagger} j$ (Sec. III in (II)) is replaced by an effective charge $Q^{e f f .}=Q\left(1-\frac{1}{6} \omega^{2}\left\langle r^{2}\right\rangle_{E}\right)$. Substituting this expression to the expression for the zero-mode part of the polarizability, Eq. (3.9), we obtain the usual retardation term, exactly as in the classical derivation of ref. 44, as well as in more general derivations [16,47].

\section{MAGNETIC POLARIZABILITY}

Although naively one would think that the analysis of the magnetic polarizabilities would parallel the electric case, it turns out that there are fundamental difficulties. The reason is the non-commutativity of the $\omega \rightarrow 0$ limit of the Compton scattering, and the large- $N_{c}$ limit, in the magnetic case. As stressed earlier, since the large- $N_{c}$ limit is the basic principle behind the hedgehog approach, one has to work consistently in the $N_{c}$ counting. We point out that with the linear response approach to the magnetic polarizabilities it is not possible. We also show importance of pionic dispersive terms in the magnetic polarizability, which enter at the same level as the pionic sea-gull contribution. Such terms were neglected in previous works [11 13.

\section{A. The $N-\Delta$ Born term}

The importance of the Born term with intermediate $\Delta$ state in the estimates for $\beta$ is a well recognized fact. The hedgehog possesses isovector magnetic moment, and the magnetic interaction term in the effective hamiltonian has the form [11, 13]

$$
H^{\text {magn. }}=3 \mu^{I=1} \boldsymbol{c} \cdot \boldsymbol{B}
$$

where $\mu^{I=1}$ is the isovector magnetic moment, and the collective vector $\boldsymbol{c}$ is defined in App. B in (II). Hamiltonian (4.1) leads to $N-\Delta$ transitions, and, according to perturbation theory, the contribution to the magnetic polarizability is

$$
\beta_{N \Delta}=18\left(\mu^{I=1}\right)^{2} \frac{\left|\left\langle N\left|c_{0}\right| \Delta\right\rangle\right|^{2}}{M_{\Delta}-M_{N}}=\frac{4\left(\mu^{I=1}\right)^{2}}{M_{\Delta}-M_{N}},
$$

where in the last equality we have used Eq. (II.B.9). Now, recalling the $N_{c}$-counting rules [19,51: $\mu^{I=1} \sim N_{c}, M_{\Delta}-M_{N} \sim 1 / N_{c}$, we immediately obtain the result

$$
\beta_{N \Delta} \sim N_{c}^{3}
$$

This surprising "superleading" behavior is the result of collective effects in the hedgehog wave function. In the orthodox approach to $N_{c}$-counting, one should stop the analysis at this point, and conclude that as far as the magnetic polarizability is concerned, one is not close to the $N_{c} \rightarrow \infty$ limit: according to Eq. (4.3) $\beta$ should be much larger than $\alpha$, which 
contradicts the experiment. Using the physical values for various magnitudes in Eq. (4.2) we obtain the numerical value

$$
\beta_{N \Delta} \sim 12 \times 10^{-4} \mathrm{fm}^{3}
$$

Note, that to compare fairly to experiment, a given model has to predict properly the value of $\mu_{N \Delta}$.

A related fundamental point is that the polarizabilities are defined in $\omega \rightarrow 0$ limits in the Compton amplitude, and in the physical world the resonances are at finite values of $\omega$, well separated from the $\omega=0$ region. In the large- $N_{c}$ limit, the $\Delta$-resonance occurs at $\omega=0$. Thus, the meaning of the polarizability is in fact lost, unless the $\omega \rightarrow 0$ limit is taken before the $N_{c} \rightarrow \infty$ limit. In the linear response method, the limits are implicitly taken in the reversed order.

In principle, a consistent analysis of the $N_{c}$-subleading physics is possible, but this would require a fully quantum-mechanical (not semiclassical) treatment, e.g. one could use the Kerman-Klein method [57]. Technically, this would be a tremendous effort, and of questionable merit in a simple effective theory such as hedgehog models.

In order to be able to make some estimates, we relax the strict $N_{c}$-counting requirement in the rest of this section, and try to examine the subleading terms in $N_{c}$ in a more "flexible" approach.

\section{B. Sea-gull term}

In hedgehog models, the magnetic seagull term is related to the electric sea-gull term by the relation (2.17). In enters at the level $N_{c}^{1}$. Numerically,

$$
\beta_{\text {sea-gull }}=-14 \times 10^{-4} \mathrm{fm}^{3},
$$

which would almost exactly cancel the $\beta_{N \Delta}$ term, in apparent rough agreement with experiment. This is, however, not the full story at the level $N_{c}$. In the next section we show, that the dispersive contributions to $\beta$ arise of the same order as $\beta_{\text {sea-gull }}$, but have positive sign, and the desired cancellation is largely suppressed.

\section{Dispersive terms}

The calculation of the magnetic dispersive terms goes along the same lines as the calculation of the electric polarizability. There are, however, a few important differences. Firstly, for the ever- $\mathcal{R} B^{1}$ perturbation the pionic term in Eq. (2.12) leads to pionic sources in the linear response equations. Since these are long-ranged (proportional to $\pi_{h}$ ), we get strong dispersive effects. The sources are listed explicitly in Table [. For the $K=0^{++}$and $2^{++}$ cases we obtain equations of the form (II.3.8). The only difference is that in the $K=0^{++}$ the valence quark eigenvalue, $\varepsilon$, changes, and the resulting quark equation in (II.3.8) has the form

$$
(h-\varepsilon) \delta q^{+}-\delta \varepsilon q_{h}-2 g M q_{h} Z=j_{q}^{+},
$$


where $\delta \varepsilon$ is the shift in the quark eigenvalue. This shift may be viewed as a Lagrange multiplier ensuring the orthogonality of the shifted quark spinor, $\delta q^{+}$, and the hedgehog quark spinor, $q_{h}$. The numerical methods of treating the $K=0^{++}$and $2^{++}$cases are straightforward.

In the $K=1^{++}$case, however, the perturbation excites the rotational zero-mode. This is a similar mechanism as in the case of the excitation of the translational zero mode in the $E^{0}$ case $-K^{\mathcal{P R}}=1^{++}$are the quantum numbers of the rotational mode in spin, or isospin (these are equivalent, since the hedgehog soliton is a $K^{\mathcal{P R}}=0^{++}$object). Thus, the hedgehog baryon placed in a constant magnetic field starts to rotate, and continues to spin up indefinitely. Quantum-mechanically, if time-dependent perturbation theory were used, it would correspond to transitions to higher spin and isospin states.

There are a few subtle issues here, concerning the order of limits. Suppose we perform (exact) projection first (e.g. suppose we have done a calculation using the Kerman-Klein method [57]), and have states with good angular momentum and isospin on which we apply the magnetic perturbation. Then, the rotational zero modes no longer appear, since they arise only if the solution breaks the symmetry of the lagrangian (Sec. III B in (II)). Thus, the appearance of the rotational zero mode in the $K^{\mathcal{P R}}=1^{++}$is the effect of starting from the unprojected hedgehog. In fact, this problem is another manifestation of the noncommutativity of the $\omega \rightarrow 0$ and the $N_{c} \rightarrow \infty$ limits, described in Sec. (IVA). One may hope, that in an improved treatment, the zero-mode physics is described by the $N-\Delta$ term, Eq. (4.2). With this in mind, in order to estimate the size of the dispersive effects, we project out the divergent zero-mode part from the $K=1$ contribution, and retain the physical part. The total result for $\beta$ consists of $\beta_{N \Delta}, \beta_{\text {sea-gull }}$, and $\beta_{\text {disp. }}$ with the zero-mode contribution projected out. As mentioned before, this is not a consistent procedure, but it allows us to estimate the importance of various effects in the magnetic polarizability.

The numerical values obtained for the $K=0,1$ and 2 parts of the $B^{1}$ magnetic perturbation are $3.6,-0.6$ and $3.3 \times 10^{-4} \mathrm{fm}^{3}$, respectively. The total dispersive contribution to $\beta_{N}$ is

$$
\beta_{\text {disp }}=6.5 \times 10^{-4} \mathrm{fm}^{3},
$$

The quark contribution is negative, and carries $-15 \%$ of the total.

The isoscalar magnetic perturbation, $B^{0}$, which is a $1 / N_{c}$ effect, and comes entirely from the quarks, contributes $0.2 \times 10^{-4} \mathrm{fm}^{3}$, which is negligible, as expected from $N_{c}$ counting. We also expect the neutron-proton splitting of magnetic polarizabilities, $\delta \beta$, to be small. It comes entirely from the quarks, since there is no magnetic analog of the electric term (2.10), and is expected to be much smaller than $\delta \alpha$, which had a pionic contribution.

At this point it might seem that adding up contributions 4.5,4.7,4.4) we still get a partial cancellation. However, the sea-gull and dispersive pieces are reduced if the pion field has the correct strength by a factor of $\sim 2$, as in the electric sea-gull case. The effects discussed in Sec. $\nabla$ further reduce these values, by another factor of 2 . These effects cause the cancellation to disappear, and we are left with a large value of $\beta_{N}$. As stressed before, rigorous estimates could only be done in a calculation beyond linear response. 


\section{ROLE OF THE $\Delta$ IN HEDGEHOG MODELS AND CHIRAL PERTURBATION THEORY}

In this section we compare the hedgehog model predictions to the results obtained in chiral perturbation theory $\chi P T$. At first, it may seem awkward, since the two methods are based on two different limits: $N_{c} \rightarrow \infty$, and $m_{\pi} \rightarrow 0$, which are known not to commute, and give different results for various observables [20]. However, as we show in Ref. [58], for quantities which are divergent in the chiral limit as $m_{\pi}^{-1}$, there is a simple connection between the hedgehog predictions and the $\chi P T$ predictions for scalar-isoscalar and vector-isovector quantities (electromagnetic polarizabilities $\alpha_{N}$ and $\beta_{N}$ are scalar-isoscalar).

Let us evaluate the polarizabilities using our methods in the chiral limit. For $\alpha_{N}$, the dominant part comes from the sea-gull term. In the chiral limit the quantity diverges as $m_{\pi}^{-1}$, and all of the contribution comes from the pionic tail, which has the form (3.2). Evaluating integral (2.17) with the profile (3.2 we obtain

$$
\alpha_{N}=\frac{5 e^{2} g_{A}^{2}}{32 \pi M^{2} m_{\pi}} .
$$

Similarly, including both the sea-gull and dispersive pieces for the magnetic polarizability, we obtain in the chiral limit

$$
\beta_{N}=\frac{e^{2} g_{A}^{2}}{64 \pi M^{2} m_{\pi}} .
$$

These expressions are exactly a factor of 3 larger than the analogous expressions following from $\chi P T$ [55]. This difference comes from the different treatment of the $\Delta$ isobar. In $\chi P T$

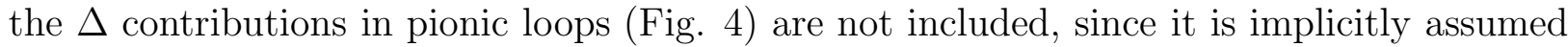
that the $N-\Delta$ mass splitting is much larger than the pion mass, and consequently loops with the $\Delta$ do not contribute to the leading singularity. They are counted as effects of order $\log \left(m_{\pi}\right), 1, \ldots$ In hedgehog models, on the contrary, the $N-\Delta$ mass splitting is a $N_{c}^{-1}$ effect, much smaller than the pion mass, which is of the order $N_{c}^{0}$. Therefore, hedgehog models include the $\Delta$ on equal footing with the nucleon. As shown below, spin and isospin Clebsch factors account for the difference by a factor of 3 between Eqs. (5.1,5.2), and $\chi P T$ predictions.

One may ask, how the physics of pionic loops, such as in Fig. 团, is present in hedgehog models. After all, the treatment of the pion field in hedgehog models is classical. To demonstrate how hedgehog expressions in the chiral limit may be viewed as hadronic loop diagrams such as in Fig. \&, let us evaluate this diagram in the chiral limit. First, we perform a calculation of the self-energy due to an insertion of the electric sea-gull interaction in the pion loop. In momentum space, we obtain

$$
\begin{aligned}
\Sigma^{*}= & \left(\frac{g_{A}}{2 F_{\pi}}\right)^{2} \int \frac{d^{4} k}{(2 \pi)^{4}} \int \frac{d^{4} q}{(2 \pi)^{4}} \tau_{a} \gamma_{5}\left(/ k+\frac{1}{2} / q\right) S_{F}(p-k) \\
& \tau_{b} \gamma_{5}\left(/ k-\frac{1}{2} / q\right) D\left(k-\frac{1}{2} q\right) \tilde{V}(q) D\left(k+\frac{1}{2} q\right) T_{a b},
\end{aligned}
$$

where $S_{F}$ is the Feynman propagator of the nucleon, $D(k)=i /\left(k^{2}-m_{\pi}^{2}+i \varepsilon\right)$ is the propagator of the pion field, $\tilde{V}(q)=\frac{1}{2} e^{2}(2 \pi)^{4} \delta^{4}(q) \frac{1}{2}(\boldsymbol{E} \cdot \nabla \boldsymbol{q})^{2}$ is the Fourier transform of the electric 
sea-gull interaction in the coordinate space, $V(\boldsymbol{r})=\frac{1}{2} e^{2}(\boldsymbol{E} \cdot \boldsymbol{r})^{2}$, and $T_{a b}=\delta_{a b}-\delta_{a 3} \delta_{b 3}$. Now we proceed as follows: we first carry out the integral over $k^{0}$ in (5.3). For the leading chiral singularity piece, $m_{\pi}^{-1}$, the contribution comes from the poles in the pionic propagators. The poles in the nucleon propagators contribute to less singular terms. We can then perform the nonrelativistic reduction of the nucleon propagator and the pion-nucleon vertices, and take the expectation value of $\Sigma^{*}$ in positive energy spinors, in order to extract the energy shift. Transforming back to coordinate space, we obtain the expression

$$
\alpha=e^{2} \int d^{3} x \phi_{a}^{a s y m \cdot}(\boldsymbol{r}) \phi_{b}^{a s y m \cdot}(\boldsymbol{r}) T_{a b},
$$

which has the form of the expression obtained in hedgehog models.

We now go back to the question of the factor of 3 . When collective coordinates are introduced, expression (5.4) becomes

$$
\alpha=e^{2} \int d^{3} x\left(\phi^{a s y m} \times \boldsymbol{c}\right)^{2} .
$$

In Sec. IIIA, to obtain the sea-gull contribution to the $\alpha_{N}$ we evaluated the collective matrix of expression (5.5) in nucleon collective wave functions. Now we repeat the procedure, but we write the resulting matrix element of the integrand in Eq. (5.5) as

$$
\sum_{i} \sum_{a}\left\langle N\left|\left(\boldsymbol{\phi}^{\text {asym. }} \times \boldsymbol{c}\right)_{a}\right| i\right\rangle\left\langle i\left|\left(\boldsymbol{\phi}^{\text {asym. }} \times \boldsymbol{c}\right)_{a}\right| N\right\rangle
$$

where $|i\rangle$ is a collective nucleon or $\Delta$ intermediate state. If the sum over $i$ is unrestricted, then we just recover our previous expressions, (5.1,5.2). If, however, we restrict $i$ to run only over nucleon collective states, then using Eq. (B10) in (II), we obtain a result smaller by a factor of 3 , and this result agrees with the $\chi P T$ prediction. Conversely, had the $\chi P T$ calculation included the $\Delta$ in diagrams of Fig. 4 , with $M_{\Delta}=M_{N}$, it would predict a result 3 times larger than quoted in Ref. [55].

Let us introduce

$$
d=\left(M_{\Delta}-M_{N}\right) / m_{\pi}
$$

These two cases, hedgehog models and $\chi P T$, correspond to two limits: $d \rightarrow 0$, and $d \rightarrow \infty$. In nature, $d \simeq 2$, which is between the two limits, and we do not have the separation of the pion mass and $M_{\Delta}-M_{N}$ scales. In this case it seems most appropriate to treat both scales as small, and keep $d$ as an unconstrained parameter. This is the spirit of the approach of Refs. [59,60]. To estimate the contribution of the $\Delta$ at the physical value of $d$, we evaluate the diagram of Fig. 4 , starting from expression (5.3), with $S_{F}$ describing the $\Delta$ propagator with the physical mass, and with the vertices modified appropriately. The $m_{\pi}^{-1}$ contribution is easily obtained, since, as in the nucleon calculation presented above, we can perform the non-relativistic reduction. The result for the ratio of the $\Delta$ to nucleon contribution in diagram 4 is

$$
\frac{\alpha^{\Delta}}{\alpha^{N}}=2 S(d),
$$

where the 2 comes from Clebsch factors, and the "mass suppression" function has the simple form 


$$
S(d)=\frac{4}{\pi} \begin{cases}\operatorname{Arctan}\left(\sqrt{\frac{1-d}{1+d}}\right) / \sqrt{1-d^{2}} & \text { for } d \leq 1 \\ \operatorname{Arctanh}\left(\sqrt{\frac{d-1}{1+d}}\right) / \sqrt{d^{2}-1} & \text { for } d>1\end{cases}
$$

For degenerate $N$ and $\Delta, S(d=0)=1$. In the limit of $d \rightarrow \infty, S(d) \sim \log d / d$. For the physical value of $d$ we find $S(2.1)=0.47$, and from Eq. (5.8) we find, that the $\Delta$ contribution in pionic loops of Fig. 1 to the electric polarizability is roughly equal to the nucleon contribution. The results for the magnetic polarizability are analogous.

This important feature of large $\Delta$ contributions in pionic loops (for scalar-isoscalar operators) is also present in other quantities [58]. In the above discussion we have tacitly assumed that the ratio $g_{\pi N \Delta} / g_{\pi N N}=3 / 2$ [20,51], as predicted by hedgehog models. Experimental numbers agree with this prediction to within a few percent. The correction may be introduced to expression (5.8) 58].

We note that the above analysis and the comparison of the hedgehog model predictions and the $\chi P T$ predictions can be done only for observables which do not depend on cranking (independent of the cranking frequency, $\lambda$ ). Observables, which do depend on cranking do not have the same chiral singularities, e.g. the electric mean squared radius diverges as $m_{\pi}^{-1}$ in hedgehog models, and as $\log m^{\pi}$ in $\chi P T$. The same is true of the splitting of electric polarizabilities, $\delta \alpha$. In this case the issue of nonocommutativity of the large- $N_{c}$ and chiral limits is much more complicated.

The results of this section indicate how one should try to improve the hedgehog predictions by subtracting the amounts by which these models overestimate the leading chiral singularity terms. There is uncertainty in such a non-rigorous procedure, but in our view it is required by physics. As already mentioned at the end of Sec. IVA, the correct treatment of the $\Delta$ could be done in a quantum calculation with the inclusion of the $N_{c}$-subleading terms.

On the other hand, the predictions of $\chi P T$ will be largely effected by the diagrams of Fig. 1 with $\Delta$ intermediate states. For polarizabilities, at the leading singularity level, these diagrams are as important as the diagrams with the nucleon intermediate states.

\section{EFFECTS OF THE PIONIC SUBSTRUCTURE}

The minimal substitution prescription in an effective lagrangian cannot produce all interactions of a hadronic system with the electromagnetic field. It is possible to write down terms which are by themselves gauge-invariant, and thus not obtainable by gauging lagrangian (2.1). In this section we focus on terms $\mathcal{L}_{9}$ and $\mathcal{L}_{10}$ of reference [50]:

$$
\begin{aligned}
\mathcal{L}_{9} & =-i L_{9} \operatorname{Tr}\left[\mathcal{F}_{\mu \nu}^{L} D^{\mu} U D^{\nu} U^{\dagger}+\mathcal{F}_{\mu \nu}^{R} D^{\mu} U^{\dagger} D^{\nu} U\right] \\
\mathcal{L}_{10} & =L_{10} \operatorname{Tr}\left[\mathcal{F}_{\mu \nu}^{L} U \mathcal{F}^{\mu \nu, R} U^{\dagger}\right]
\end{aligned}
$$

where $\mathcal{F}_{\mu \nu}^{L, R}$ are the left and right chiral field strength tensors. For the case of electromagnetic field we have $\mathcal{F}_{\mu \nu}^{L, R}=e \frac{1}{2} \tau_{3} F_{\mu \nu}=e \frac{1}{2} \tau_{3}\left(\partial_{\mu} A_{\nu}-\partial_{\nu} A_{\mu}\right)$. In the linear $\sigma$-model, $U$ corresponds describes the chiral field, $U=F_{\pi}^{-1}(\sigma+i \boldsymbol{\tau} \cdot \boldsymbol{\pi})$. The constants $L_{9}$ and $L_{10}$ can be expressed through measurable quantities, namely the pion electric mean square radius, $\left\langle r^{2}\right\rangle_{E}^{\pi}$, and the pion polarizability $\bar{\alpha}^{\pi}=-\bar{\beta}^{\pi}[50,49]$ : 


$$
\begin{aligned}
L_{9} & =\frac{F_{\pi}^{2}\left\langle r^{2}\right\rangle_{E}^{\pi}}{12}, \\
L_{10} & =\frac{m_{\pi} F_{\pi}^{2} \bar{\alpha}^{\pi}}{4}-L_{9}=\frac{m_{\pi} F_{\pi}^{2} \alpha^{\pi}}{4} .
\end{aligned}
$$

In the chiral perturbation theory treatment, one considers pionic loops, and through renormalization $L_{9}$ and $L_{10}$ acquire chiral logarithms. In our approach, we simply treat the terms (6.2) in the mean-field approximation, replacing the meson field operators by classical fields. As before, the $E$ and $B$ fields are constant, and we find from the $\mathcal{L}_{9}$ term

$$
\begin{aligned}
\int d^{3} x \mathcal{L}_{9} & =4 e^{2} L_{9} F_{\pi}^{-2} \int d^{3} x F^{i \nu} A_{\nu}\left(\boldsymbol{c} \times \boldsymbol{\pi}_{h}\right) \cdot\left(\boldsymbol{c} \times \partial_{i} \boldsymbol{\pi}_{h}\right) \\
& =2 L_{9}\left(E^{2}-B^{2}\right) \int d^{3} x\left(\boldsymbol{c} \times \boldsymbol{\pi}_{h}\right)^{2},
\end{aligned}
$$

where $i$ runs over spatial values. The $\mathcal{L}_{10}$ term leads to

$$
\begin{aligned}
\int d^{3} x \mathcal{L}_{10}= & 4 e^{2} L_{10}\left(E^{2}-B^{2}\right) F_{\pi}^{-2} \\
& \int d^{3} x\left(\left(\boldsymbol{c} \times \boldsymbol{\pi}_{h}\right)^{2}-\frac{1}{2}\left(\sigma_{h}^{2}+\boldsymbol{\pi}_{h}^{2}\right)\right) .
\end{aligned}
$$

The last term under the integrand is canceled by the vacuum subtraction, which is implicit. It becomes $-\left(\sigma_{h}^{2}+\boldsymbol{\pi}_{h}^{2}\right)+\sigma_{v a c}^{2}=-\left(\sigma_{h}^{2}+\boldsymbol{\pi}_{h}^{2}\right)+F_{\pi}^{2}$, which is zero in the nonlinear sigma model, but also vanishingly small in our case due to proximity of the hedgehog solution to the chiral circle. We can thus drop this term in our estimate. Using (6.4, II.3.21) we read off from (6.5, 6.6) the following contribution to the nucleon polarizabilities:

$$
\alpha_{N}^{\pi}=-\beta_{N}^{\pi}=m_{\pi} \Theta^{m e s} \bar{\alpha}^{\pi} .
$$

The physical interpretation of this contribution is clear. The pion, having electromagnetic structure, is polarizable. Since the nucleon is surrounded by a pion cloud, this pion polarizability results in additional polarizability of the nucleon. Note the opposite signs of the electric and magnetic polarizabilities in (6.7), reflecting the fact that $\overline{\alpha^{\pi}}=-\overline{\beta^{\pi}}$. Also notice, that since $\bar{\alpha}^{\pi}$ is of order 1 in $N_{c}$-counting, the contributions 6.7 are of order $N_{c}$. The terms (6.2) do not lead to additional neutron-proton splitting of polarizabilities

We can call $N_{\pi}=m_{\pi} \Theta^{\text {mes }}$ in 6.7 the "number of pions" in the nucleon seen in the Compton scattering process. Numerically, $N_{\pi} \simeq 0.5$ in our model. Using the relation of the moment of inertia to the $N-\Delta$ mass splitting, we can write

$$
N_{\pi}=\frac{3}{2} \frac{m_{\pi}}{M_{\Delta}-M_{N}} \frac{\Theta^{m e s}}{\Theta},
$$

where the last factor is the fraction of the total moment of inertia carried by the pion. This quantity is $\sim 1$ in hedgehog models: $60 \%$ in our model with quarks, $100 \%$ in the Skyrmion. We thus have a quasi-model-independent result $N_{\pi} \sim 0.5-0.7$.

The value of $\alpha^{\pi}$ can be determined experimentally, however existing experimental data 61] do not seem reliable, and are in contradiction with a low-energy theorem due to Holstein [49], which gives $\alpha^{\pi}=2.8 \times 10^{-4} \mathrm{fm}^{3}$. With this value we get 


$$
\alpha_{N}^{\pi}=-\beta_{N}^{\pi} \sim 1.3 \times 10^{-4} \mathrm{fm}^{3}
$$

which is a few times smaller compared to the minimal substitution terms, but non-negligible, especially for the magnetic case, where we expect cancellations to occur. If experimental numbers for $\alpha^{\pi}$ were used [61], then a three times larger result would follow.

Other non-minimal substitution terms can also be considered, but there is no knowledge of the phenomenological low-energy constants which have to be introduced, and predictive power is lost.

\section{OTHER MODELS}

The analysis of this paper can be straightforwardly applied to other chiral models. In purely mesonic Skyrmions [21], the RPA approximation corresponds to linearizing the small fluctuation equations, hence the non-linearity constraint is not imposed at the quantum level. Appropriate linear response equations can be derived along the lines of App. B in (II). The role of the isoscalar source is carried by the topological (Goldstone-Wilczek) current, and effects of the higher order terms in the equations are present.

In purely quark models (NJL) [32], upon minimal substitution, the lagrangian in presence of the electromagnetic field $A^{\mu}$, and after introducing collective degrees of freedom, becomes

$$
\begin{aligned}
S_{N J L}\left(A^{\mu}\right) & =-i \operatorname{Tr} \log \left(i / \partial-g\left(\sigma+i \gamma_{5} \boldsymbol{\tau} \cdot \boldsymbol{\pi}\right)-\frac{1}{2} \gamma_{0} \boldsymbol{\tau} \cdot \boldsymbol{\lambda}\right. \\
& \left.+\frac{1}{2} / A\left(N_{c}^{-1}+\boldsymbol{\tau} \cdot \boldsymbol{c}\right)\right)-(v a c)
\end{aligned}
$$

where $(v a c)$ denotes the vacuum subtraction, and an NJL cut-off is understood. The trace accounts for the occupied (valence) levels. This expression may be expanded to second-order in $A^{\mu}$, and expressions for polarizabilities can be easily derived. The dispersive pieces, as in the model treated in this paper, lead in case of even- $\mathcal{R}$ interactions to distortions in the profile functions $\sigma$ and $\boldsymbol{\pi}$. This is likely to result in numerical complications, in particular in cases with zero-mode excitations. One can use gradient expansion techniques [62] instead of solving the model exactly. Then, for example, the isovector electric responce of the quarks from the Dirac sea generates the pionic sea-gull contribution.

All our generic hedgehog model conclusions described in this paper hold for these, and other hedgehog models.

\section{CONCLUSION}

The main results obtained in this paper can be summarized as follows:

1) We show the $N_{c}$-counting rules for electromagnetic polarizabilities in hedgehog models. For electric polarizabilities the basic experimental pattern is reproduced $\left(\alpha_{N} \sim N_{c}>\delta \alpha \sim\right.$ $\left.1 / N_{c}\right)$. For the magnetic case the rules show the inapplicability of linear response $\left(\beta_{N} \sim N_{c}^{3}\right)$.

2) Dispersive terms lead to deformation of hedgehog solitons, and the resulting contributions to polarizabilities enter at the same $N_{c}$-level as the sea-gull contributions. In the magnetic case, we expect that the presence of dispersive terms leads to large positive contributions to $\beta_{N}$. There is no cancellation mechanism which can bring $\beta$ down to the 
experimental value. In fact, all model calculations with the $\Delta$ degree of freedom have this problem, since the paramagnetic $N-\Delta$ term is large, and there is no simple mechanism to cancel this effect.

3) Hedgehog models provide a mechanism of splitting of the neutron and proton polarizabilities. An explicit calculation gives reasonable numbers, and the sign of $\delta \alpha=\alpha_{n}-\alpha_{p}$ is expected to be positive in models with pionic clouds.

4) Concerning numerical results in hedgehog models, because of the sensitivity of the results to the pion tail, the value of $g_{A}$ in a model should be well reproduced. Also, for the magnetic case, good prediction for $\mu_{N \Delta}$, as well as $M_{\Delta}-M_{N}$, is necessary to reproduce the $N-\Delta$ paramagnetic term.

5) The $\Delta$ resonance plays an important role. Hedgehog models largely overestimate these contributions, since they neglect the effects of $N-\Delta$ mass splitting in projection. We show how to estimate the $\Delta$ effects in pionic loops in a modified chiral perturbation theory, and find these effects are at the level of $100 \%$ in calculation of the electromagnetic polarizabilities. One should also try to improve the hedgehog predictions by subtracting the amounts by which these models overestimate the leading chiral singularity terms.

6) The effects of non-minimal substitution terms $\mathcal{L}_{9}$ and $\mathcal{L}_{10}$ [50] in the effective lagrangian enter at the level of $1-2 \times 10^{-4} \mathrm{fm}^{3}$.

\section{ACKNOWLEDGMENTS}

Support of the the National Science Foundation (Presidential Young Investigator grant), and of the U.S. Department of Energy is gratefully acknowledged. We thank Manoj Banerjee for many useful suggestions and countless valuable comments. One of us (WB) acknowledges

a partial support of the Polish State Committee for Scientific Research (grants 2.0204.91.01 and 2.0091.91.01). 


\section{REFERENCES}

* On leave of absence from H. Niewodniczański Institute of Nuclear Physics, ul. Radzikowskiego 152, 31-342 Cracow, POLAND.

[1] F. Federspiel et al., Phys. Rev. Lett. 67, 1511 (1991).

[2] A. Zieger et al., Phys. Lett. B 278, 34 (1992).

[3] J. Schmiedmayer, P. Riehs, J. Harvey, and N. Hill, Phys. Rev. Lett. 66, 1015 (1991).

[4] For a review of experiments on electric polarizability see for example J. Schmiedermayer, H. Rauch, and P. Riehs, Nucl. Instrum. Methods. Phys. Res. A 284, 137 (1989).

[5] For a review of earlier calculations in quark models see G. Dattoli, G Matone and D. Prosperi, Lett. Nuovo Cimento 19, 601 (1977).

[6] P. Hecking and G. F. Bertsch, Phys. Lett. B 99, 237 (1981).

[7] H. Krivine and J. Navarro, Phys. Lett. B 171, 331 (1986).

[8] A. Schäfer, B. Müller, D. Vasak, and W. Greiner, Phys. Lett. B 143, 323 (1984).

[9] S. Capstick and B. D. Keister, Technical Report CEBAF-TH-91-22, CEBAF, 1991.

[10] R. Weiner and W. Weise, Phys. Lett. 159B, 85 (1985).

[11] E. M. Nyman, Phys. Lett. 142B, 388 (1984).

[12] M. Chemtob, Nucl. Phys. A473, 613 (1987).

[13] N. N. Scoccola and W. Weise, Phys. Lett. B232, 495 (1989); Nucl. Phys. A517, 495 (1990).

[14] H. R. Fiebig, W. Wilcox, and R. M. Woloshyn, Nucl. Phys. B 324, 47 (1989).

[15] V. A. Petrun'kin, Sov. J. Part. Nuc. 12, 278 (1981).

[16] J. L. Friar, in Proc. Workshop on electron-nucleus scattering, EIPC, Marciana, Italy, edited by A. Fabrocini et al., World Scientific, Singapore, 1989.

[17] W. Broniowski and T. D. Cohen, Technical Report DOE/ER/40322-155, U. of MD PP \# 92-193, Univ. Of Maryland, 1992, The following article.

[18] T. H. R. Skyrme, Proc. R. Phys. Soc. London A260, 127 (1961); Nucl. Phys. 31, (1962) 556.

[19] G. S. Adkins, C. R. Nappi, and E. Witten, Nucl. Phys. B228, 552 (1983).

[20] G. S. Adkins and C. R. Nappi, Nucl. Phys. B233, 109 (1984).

[21] I. Zahed and G. E. Brown, Phys. Rep. 142, 1 (1986), and references therein.

[22] M. C. Birse and M. K. Banerjee, Phys. Lett. 136B, 284 (1984); Phys. Rev. D 31, 118 (1985).

[23] G. R. S. Kahana and V. Soni, Nucl. Phys. A415, 351 (1984).

[24] S. Kahana and G. Ripka, Nucl. Phys. A419, 462 (1984).

[25] G. Kalberman and J. M. Eisenberg, Phys. Lett. 139B, 337 (1984).

[26] M. K. Banerjee, W. Broniowski, and T. D. Cohen, in Chiral Solitons, edited by K.-F. Liu, World Scientific, Singapore, 1987.

[27] M. C. Birse, Progress in Part. and Nucl. Physics 25, 1 (1990).

[28] L. Vepstas and A. D. Jackson, Phys. Rep. 187, 109 (1990), and references therein.

[29] M. K. Banerjee, in Proc. of Workshop on Nuclear Chromodynamics, Santa Barbara, 1985, edited by S. Brodsky and E. Moniz, World Scientific, Singapore, 1985.

[30] W. Broniowski, Fizika 19 Supplement 2, 38 (1987), Proceedings of Workshop on Mesonic Degrees of Freedom in Nuclei, Bled/Ljubljana, Yugoslavia, 1987.

[31] I. Duck, Phys. Rev. D 34, 1493 (1986).

[32] Y. Nambu and G. Jona-Lasinio, Phys. Rev. 122, 345 (1961). 
[33] D. I. Dyakonov and V. Y. Petrov, JETP Lett. 43, 57 (1986).

[34] D. I. Dyakonov, V. Yu. Petrov, and P. V. Pobylitsa, Nucl. Phys. B306, 809 (1988).

[35] Th. Meissner, E. Ruiz Arriola, F. Grümer, K. Goeke and H. Mavromatis, Phys. Lett. B214, 312 (1988).

[36] H. Reinhardt and R. Wünsch, Phys. Lett. B215, 577 (1988).

[37] Th. Meissner, F. Grümer and K. Goeke, Phys. Lett. B227, 296 (1989).

[38] D. I. Dyakonov, V. Yu. Petrov, and M. Praszałowicz, Nucl. Phys. B323, 53 (1989).

[39] R. Alkofer, Phys. Lett. B236, 310 (1990).

[40] G. 't Hooft, Nucl. Phys. B75, 461 (1974).

[41] E. Witten, Nucl. Phys. B160, 57 (1979).

[42] W. Broniowski, M. K. Banerjee, and T. D. Cohen, Technical Report DOE/ER/40322144, U. of MD PP \# 92-130, Univ. Of Maryland, 1991, To appear in Phys. Lett. B.

[43] Strictly speaking, this definition is for charge-neutral particles only, but can be easily modified for the case of charged objects (see discussion in Sec. IIIC.

[44] T. E. O. Ericson and J. Hüfner, Nucl. Phys. B57, 808 (1961).

[45] A. M. Baldin, Nucl. Phys. 18, 310 (1960).

[46] A. Klein, Phys. Rev. 99, 998 (1955).

[47] V. A. Petrun'kin, Sov. Phys. JETP 13, 278 (1981).

[48] The error estimates are from a talk by A. Nathan presented at the Baryons '92 conference, New Haven, June 1-4, 1992.

[49] B. R. Holstein, Comments Nucl. Part. Phys. A 19, 221 (1990).

[50] J. Gasser and H. Leutwyler, Ann. Phys. (N.Y.) 158, 142 (1989).

[51] T. D. Cohen and W. Broniowski, Phys. Rev. D34, 3472 (1986).

[52] M. Bando, T. Kugo, and K. Yamawaki, Phys. Reports 164, 217 (1988).

[53] W. Broniowski and M. K. Banerjee, Phys. Lett. 158B, 335 (1985); Phys. Rev. D 34, 849 (1986).

[54] Note that vector mesons acquire shifts also upon cranking, which is an odd grandreversal perturbation: W. Broniowski and T. D. Cohen, Phys. Lett. 177B, 141 (1986).

[55] V. Bernard, N. Kaiser, and U.-G. Meissner, Phys. Rev. Lett. 67, 1515 (1991); Nucl. Phys. B 373, 346 (1992).

[56] U. E. Schröder, Nucl. Phys. B166, 103 (1980).

[57] A. Kerman and A. Klein, Phys. Rev. 132, 1326 (1963). For soliton models this method was introduced by J. Goldstone and R. Jackiw, Phys. Rev. D 11, 1486 (1975).

[58] T. D. Cohen and W. Broniowski, Technical Report DOE/ER/40322-154, U. of MD PP \# 92-191, Univ. of Maryland, 1992.

[59] E. Jenkins and A. Manohar, Phys. Lett. B255, 558 (1991); E. Jenkins, Nucl. Phys. B368, 190 (1992).

[60] E. Jenkins and A. Manohar, Technical Report UCSD/PTH 91-30, 1991.

[61] For references see Ref. [49].

[62] See Refs. (49-51) in (II). 


\section{FIGURES}

FIG. 1. Dispersive contributions to the Compton scattering amplitude. In our treatment, $\mathcal{G}$ is the RPA (linear response) propagator.

FIG. 2. Radial densities of the leading- $N_{c}$ contributions to $\alpha_{N}$ (in units of $10^{-4} \mathrm{fm}^{2}$ ): Sea-gull (solid line), and quark $E^{1}$ contribution (dashed line). The dotted line shows the sea-gull term evaluated with the asymptotic profile, Eq. (3.2).

FIG. 3. Radial density of the pion contribution to the splitting of the neutron and proton electric polarizabilities, $\delta \alpha$ (in units of $10^{-4} \mathrm{fm}^{2}$ ).

FIG. 4. Hadronic one-pion-loop diagrams giving the leading chiral contribution to nucleon

polarizabilities. The vertex corresponds to the sea-gull interaction $e^{2} \int d^{3} x(\boldsymbol{E} \cdot \boldsymbol{x})(\boldsymbol{c} \times \boldsymbol{\pi})$ 


\section{TABLES}

TABLE I. $K^{P R}$ classification of various dispersive perturbations, and sources in the corresponding linear response equations (App. A in (II)). Quantum numbers $(L, \Lambda)$ label quark shifts, $L_{\pi}$ and $L_{\sigma}$ are the orbital numbers of meson shifts. Null entry in columns for $L_{\pi}$ and $L_{\sigma}$ means that the fluctuation does not arise. For cases with zero modes (electric isoscalar and magnetic isovector, $\mathrm{K}=1$ ), the sources listed are twice the $j_{X}, j_{Y}$ or $j_{Z}$ sources from equations in App. A in (II). For other cases they are the sources entering Eqs. (II.3.7,II.3.8). Radial functions $G_{h}$ and $F_{h}$ are the upper and lower components of the hedgehog valece spinor, and $\pi_{h}$ in the hedgehog pion profile. See (II) for details.

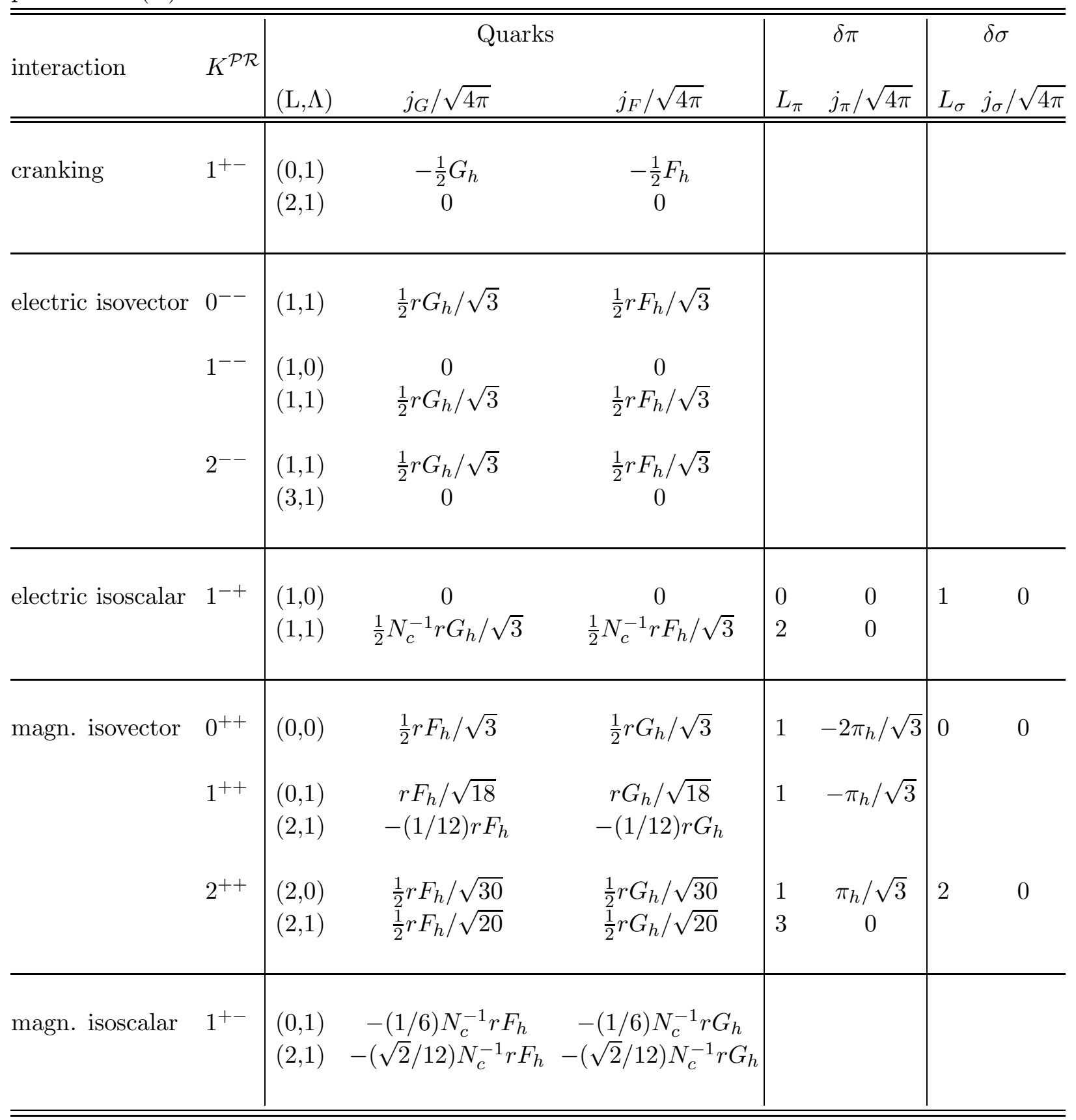


TABLE II. Electric polarizability. The model predictions for $\alpha_{N}$ are expected to be largly reduced by effects disussed in the text.

\begin{tabular}{|c|c|}
\hline \multicolumn{2}{|c|}{$\alpha_{N}\left(10^{-4} \mathrm{fm}^{3}\right)$} \\
\hline sea-gull & 28.5 \\
\hline dispersive $E^{1}$ (quarks) & 3.5 \\
\hline total & 32 \\
\hline experiment & $9.6 \pm 1.8 \pm 2.2$ \\
\hline \multicolumn{2}{|c|}{$\delta \alpha\left(10^{-4} \mathrm{fm}^{3}\right)$} \\
\hline$\overline{\text { pion }}$ & 5.6 \\
\hline quarks & -0.2 \\
\hline total & 5.4 \\
\hline experiment & $4.8 \pm 1.8 \pm 2.2$ \\
\hline
\end{tabular}

